\title{
Evolution of neutrophil apoptosis in septic shock survivors and nonsurvivors 2 放, 放放
}

\author{
Eduardo Tamayo MD, PhD ${ }^{\text {a,1 }}$, Esther Gómez MD, PhD ${ }^{a, 1}$, \\ Juan Bustamante MD, PhD ${ }^{b, *, 1}$, José I. Gómez-Herreras MD, PhD ${ }^{c, 1}$, \\ Rosalba Fonteriz MD, PhD ${ }^{d, 1}$, Felipe Bobillo MD, PhD ${ }^{e, 1}$, \\ Jesús F. Bermejo-Martín MD, PhD ${ }^{f, 1}$, Javier Castrodeza MD, PhD ${ }^{g, 1}$, \\ Maria Heredia $M D^{a, 1}$, Inma Fierro $M D^{h, 1}$, Francisco Javier Álvarez MD, PhD ${ }^{h, 1}$
}

${ }^{a}$ Department of Anaesthesiology and Intensive Care, Hospital Clínico Universitario de Valladolid, 47005 Valladolid, Spain

${ }^{\mathrm{b}}$ Department of Cardiovascular Surgery. Hospital Universitario La Princesa, 28006 Madrid, Spain

${ }^{\mathrm{c}}$ Department of Anaesthesiology and Intensive Care, Hospital Universitario Rio Hortega, 47012 Valladolid, Spain

${ }^{\mathrm{d}}$ Institute of Biology and Molecular Genetics (IBGM/CSIC), Universidad de Valladolid, 47005 Valladolid, Spain

${ }^{\mathrm{e}}$ Medical Intensive Care Unit, Hospital Clínico Universitario de Valladolid, 47005 Valladolid, Spain

${ }^{\mathrm{f}}$ Infection \& Immunity Unit, Hospital Clinico Universitario de Valladolid -IECSCYL, 47005 Valladolid, Spain

${ }^{\mathrm{g}}$ Direction of Public Health, Investigation, Development and Innovation, SACYL, 47007 Valladolid, Spain

${ }^{\mathrm{h}}$ Department of Pharmacology and Therapeutics, Facultad de Medicina, Universidad de Valladolid, 47005 Valladolid, Spain

\section{Keywords: \\ Apoptosis; \\ Neutrophil; \\ Septic shock; \\ Mortality; \\ Survivors}

\begin{abstract}
Purpose: The aims were to analyze the temporal evolution of neutrophil apoptosis, to determine the differences in neutrophil apoptosis among 28-day survivors and nonsurvivors, and to evaluate the use of neutrophil apoptosis as a predictor of mortality in patients with septic shock.

Materials and Methods: Prospective multicenter observational study carried out between July 2006 and June 2009. The staining solution study included 80 patients with septic shock and 25 healthy volunteers. Neutrophil apoptosis was assessed by fluorescein isothiocyanate (FITC)-conjugated annexin V and aminoactinomycin D staining.

Results: The percentage of neutrophil apoptosis was significantly decreased at 24 hours, 5 days, and 12 days after the diagnosis of septic shock $(14.8 \% \pm 13.4 \%, 13.4 \% \pm 8.4 \%$, and $15.4 \% \pm 12.8 \%$, respectively; $P<.0001)$ compared with the control group $(37.6 \% \pm 12.8 \%)$. The difference in apoptosis between 28 -day surviving and nonsurviving patients was nonsignificant $(P>.05)$. The mortality rate at 28 days was $53.7 \%$.
\end{abstract}

\footnotetext{
Conflict of Interest: None declared.

放放 Funding Sources: This work was supported in part by a grant from the "Gerencia de Salud, Consejería de Sanidad, Junta de Castilla y Leon" (GRS 143/ A/07) and Instituto de Salud Carlos III, Fondo Europeo de Desarrollo Regional FEDER, Red de Trastornos Adictivos RD06/0001/0020.

* Corresponding author. Tel.: +34 915202268; fax: +34 915202201.

E-mail addresses: tamayo@med.uva.es (E. Tamayo), egomez@hotmail.com (E. Gómez), jgomez012001@yahoo.es (J.I. Gómez-Herreras), bustamj@hotmail.com, jbustamantemunguira@gmail.com (J. Bustamante), fonteriz@med.uva.es (R. Fonteriz), bobillo@med.uva.es (F. Bobillo), castrodeza@med.uva.es (J. Castrodeza), heredia@hotmail.com (M. Heredia), inma@lti.uva.es (I. Fierro), alvarez@med.uva.es (F.J. Álvarez).

${ }^{1}$ Valladolid Sepsis Study Group.
} 
The crude hazard ratio for mortality in patients with septic shock did not differ according to the percentage of apoptosis (hazard ratio, 1.006; 95\% confidence interval, $0.98-1.03 ; P=.60$ ).

Conclusions: During the first 12 days of septic shock development, the level of neutrophil apoptosis decreases and does not recover normal values. No differences were observed between surviving and nonsurviving patients.

(C) 2012 Elsevier Inc. All rights reserved.

\section{Introduction}

Sepsis affects more than 500000 patients annually in the United States [1], with an estimated incidence in Europe of 240 to 400 cases per 100000 inhabitants per year [2], and the incidence rate continues to increase [1,2]. Of the patients admitted to intensive care units (ICUs), the incidence of severe sepsis or septic shock is between $9 \%$ and $37 \%$ [1-4]. In spite of recent advances in treatment with antibiotics and critical care therapy, sepsis still results in a high mortality rate; it is typically between $40 \%$ and $60 \%$ in the case of septic shock [1-5]. Septic shock and multiple organ dysfunction syndrome (MODS) are the most common causes of death in ICUs [4].

The lack of apoptosis regulation in patients with severe injuries contributes to the pathogenesis of MODS [6]. The systemic inflammatory response can harm the patient through either an inadequate or excessive inflammatory reaction [7]. Apoptosis is vital to the regulation of neutrophils, monocyte eosinophils, and macrophages. With sepsis, a delay in neutrophil apoptosis [8] and increased apoptosis in hematopoietic tissues, such as the thymus, Peyer patch, spleen, and bone marrow, is frequently observed [9]. The prolonged neutrophil survival may allow a more robust inflammatory response and, thus, better protect the individual. However, neutrophil survival may also cause harm to the host, leading to the development of MODS and increasing mortality by increasing the number of leukocytes or the production of proinflammatory mediators and free radicals [10-12].

Although the significant reduction in neutrophil apoptosis has been sufficiently established in patients with sepsis $[8,10]$, the temporal course of this phenomenon during the clinical evolution of septic shock has not been studied in either surviving or nonsurviving patients. The use of neutrophil apoptosis as a predictor of survival in patients with septic shock has also not been studied.

Based on the fact that the reduction in neutrophil apoptosis during septic shock is related to the development of MODS [6,10,11], we hypothesize that the levels of neutrophil apoptosis in patients who survive septic shock will continue to increase throughout its evolution until normal levels, values equivalent to those found in healthy controls, are reached, and these levels will be different (ie, higher) than those in nonsurviving patients.

The aim of this study was to analyze the following in patients with septic shock: (1) temporal changes in neutrophil apoptosis, (2) differences in neutrophil apoptosis between 28-day survivors and 28-day nonsurvivors, (3) the use of neutrophil apoptosis as a predictor of mortality, and (4) evaluation of the factors that may influence the course of the apoptosis.

\section{Materials and methods}

\subsection{Study design, patients, and healthy volunteers}

From July 2006 through June 2009, eligible patients were enrolled in this open-label, prospective, nonrandomized, multicenter, nondrug interventional study, which was conducted at $4 \mathrm{medical} / \mathrm{surgical}$ ICUs in Valladolid, Spain: postoperative general, postoperative cardiac surgery, and medical ICUs of the Hospital Clinico Universitario and the postoperative general ICU of the Hospital Universitario Río Hortega. The study protocol was reviewed and approved by the Human Subjects Review Committee and Research Ethics Board of the Hospital Clínico Universitario of Valladolid, and informed consent was obtained from each study participant.

The study only included patients older than 18 years who were in septic shock with at least 2 dysfunctional organs or systems at the time of enrollment, as previously described by Bone et al [7] (Appendix A). The patients were enrolled in the first 24 hours after the diagnosis of septic shock. Patients were excluded if they had a known metastatic malignant disease or HIV, were receiving immunosuppressive drugs or systemic corticosteroids, or were enrolled in a concomitant interventional study.

Over the course of the study, a total of 92 patients met the criteria for inclusion; however, 12 patients had incomplete follow-up and were withdrawn from the study. In total, the study included 80 patients and 25 healthy volunteers, who were included as normal controls (Appendix B). The 80 septic shock patients had a mean age $( \pm \mathrm{SD})$ of $68.7 \pm 12.4$ years and consisted of 52 men $(65.0 \%)$ and 28 women (35.0\%). The mean age $( \pm \mathrm{SD})$ of the 25 healthy controls was $68.5 \pm 17.3$ years and consisted of 15 men $(60.0 \%)$ and 10 women $(40.0 \%)$. No significant differences were observed between the 2 groups with respect to age or sex $(P>.05)$.

The septic shock patients included in the study were divided into 2 groups: 28-day nonsurvivors and 28-day survivors (Appendix B). A follow-up period of 12 days was established for the evolution of apoptosis based on data from previous studies concerning the duration of stay in the hospital and ICU. Rivers et al [13] reported a mean hospital 
stay of $13.0 \pm 13.7$ days; Angus et al [1] reported a mean ICU stay of $15.7 \pm 7$ days; Esteban et al [2] reported a mean ICU stay of 9 days (range, 4-25 days); and Ferreira et al [14] reported a mean ICU stay of 4.0 days (range, 1-56 days).

Patients were treated according to the best standard of care for severe sepsis. The therapeutic measures were those habitually used in the ICUs in which the study was conducted.

\subsection{Blood samples, neutrophil isolation, and neutrophil apoptosis}

\subsubsection{Blood samples}

A blood sample of $10 \mathrm{~mL}$ was obtained from each patient immediately after providing informed consent (24 hours), at 5 days, and at 12 days. A single peripheral blood extraction of $10 \mathrm{~mL}$ was obtained from each healthy control. The blood samples were placed in tubes containing citrate and glucose, and the samples were sent to the laboratory of the Valladolid Institute of Biology and Molecular Genetics for analysis.

\subsubsection{Isolation of neutrophils}

Neutrophils were isolated as described previously [14]. Fresh blood was mixed 6:1 (vol/vol) with acid-citrate-dextrose. Dextran (T500 Pharmacy) (Pharmacosmos A/S, Holbaek, Denmark) was added to a final concentration of $1.3 \%$. After 45 minutes at room temperature, the upper layer containing no red blood cells was centrifuged ( $300 \mathrm{~g}, 10$ minutes). The cell pellet was resuspended and layered on a Ficoll gradient (Lymphocyte Separation Medium; Flow Laboratories, Irvine, UK). After 40 minutes of centrifugation $(400 \mathrm{~g})$, the pellet containing neutrophils was recovered and contaminating red cells disrupted by hypotonic lysis. Neutrophils were resuspended in 1-mL phosphate-buffered saline and counted by trypan blue exclusion. Viability was more than $95 \%$. To confirm cell viability, we measured cytosolic calcium in parallel experiments. Cells were able to respond with calcium increases in response to fMet-Leu-Phe: formal-methionyl-leucyl-phenylalanine and platelet-activating factor.

\subsection{Neutrophil apoptosis}

Neutrophil apoptosis was detected using a technique combining fluorescein isothiocyanate (FITC)-conjugated annexin V (annexin V-FITC) and 7-aminoactinomycin D (7ADD) (Molecular probes; Invitrogen; Paisley, Renfrewshire, UK) staining solution (Annexin V-FITC Apoptosis Detection kit I; BD PharMingen, San Diego, CA). The polymorphonuclear suspension $(100 \mu \mathrm{L})$ in cold binding buffer $(200000$ cells $/ \mathrm{mL})$ was transferred to 4 culture tubes to which $5 \mu \mathrm{L}$ of annexin $\mathrm{V}$ (BD PharMingen) was added. After incubation in the dark at room temperature for 15 minutes, $400 \mu \mathrm{L}$ of annexin $\mathrm{V}$-binding buffer (BD PharMingen) and $0.4 \mu \mathrm{L}$ of a $1 \mathrm{mg} / \mathrm{mL}$ stock of 7ADD were added to each tube for flow cytometry (FACSCalibur; Becton Dickinson, Fullerton, CA). Gating based on forward and side-scatter detection was applied to eliminate cell debris. A minimum of 10000 events were acquired per sample.
Gated events were plotted for annexin V-FITC and 7-ADD staining. Results were reported as the percentage of viable cells, cells in the early stages of apoptosis (annexin V-FITC positive and 7-ADD negative), or dead cells (Fig. 1). All the experiments were made in by duplicate with controls with only 7-ADD and only annexin $\mathrm{V}$ to perform compensation.

We carried out a morphological control of apoptosis in parallel experiments: neutrophils were stained with MayGrünwald-Giemsa; the cells that showed shrinkage, nuclear coalescence, and nuclear chromatin condensation were considered as apoptotic; the number was very similar to annexin V results: apoptosis values were very different ranging from $13.8 \% \pm 2.1 \%$ in septic patients to $46.7 \pm 3.7$ in control neutrophils (Fig. 1B) [15].

\subsection{Study variables}

\subsubsection{Outcome variables}

The primary outcome variable was the mean percentage of neutrophil apoptosis.

\subsubsection{Independent variables}

Independent variables included age, sex, prior or preexisting conditions (hypertension, coronary artery disease, other heart disease, congestive heart failure, diabetes mellitus, chronic pulmonary disease, liver disease, cancer, chronic renal failure), admission category (medical, elective surgery, emergency surgery, type surgery), severity of septic shock (Acute Physiology and Chronic Health Evaluation [APACHE] II score [16]; Sequential Organ Failure Assessment [SOFA] score [17]; acute respiratory distress syndrome; lactate; C-reactive protein [CRP]; central venous oxygen saturation $\left[\mathrm{ScvO}_{2}\right]$; procalcitonin; vasopressor use; time to extubation; leukocyte count measured at 24 hours, 5 days, and 12 days; length of hospital stay; and length of ICU stay, 28-day mortality, and in-hospital mortality from the time of sepsis diagnosis), specific strategies for the pharmacologic treatment of sepsis (insulin therapy directed at maintaining a blood glucose level $<150 \mathrm{mg} / \mathrm{dL}$ blood, lowdose steroid therapy, activated protein $\mathrm{C}$ therapy, appropriate antibiotic therapy), site of infection, and culture results on the day of the study. Appropriate empiric antibiotic treatment was defined when at least one of the drugs administered was effective against the pathogens obtained on the antibiogram and administered immediately after the microbiological diagnostic test and continued for at least 5 days or until death. The only exception was the case of Pseudomonas aeruginosa, in which 2 effective drugs were required.

\subsection{Determination of sample size}

The sample size calculation was based on the percentage of neutrophil apoptosis in healthy volunteers, as opposed to that of patients with septic shock, from published studies. At the start of the study (at 24 hours), the frequency of neutrophil apoptosis in patients with septic shock was 


\section{Patient with sepsis (A)}

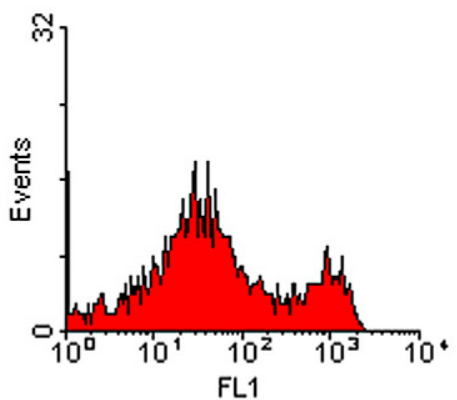

Annexin-V FFITC

Healthy volunteer (B)

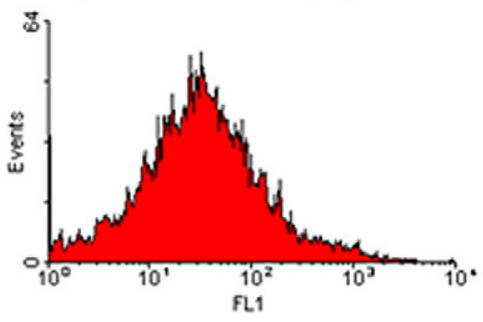

Annexin-V FFITC
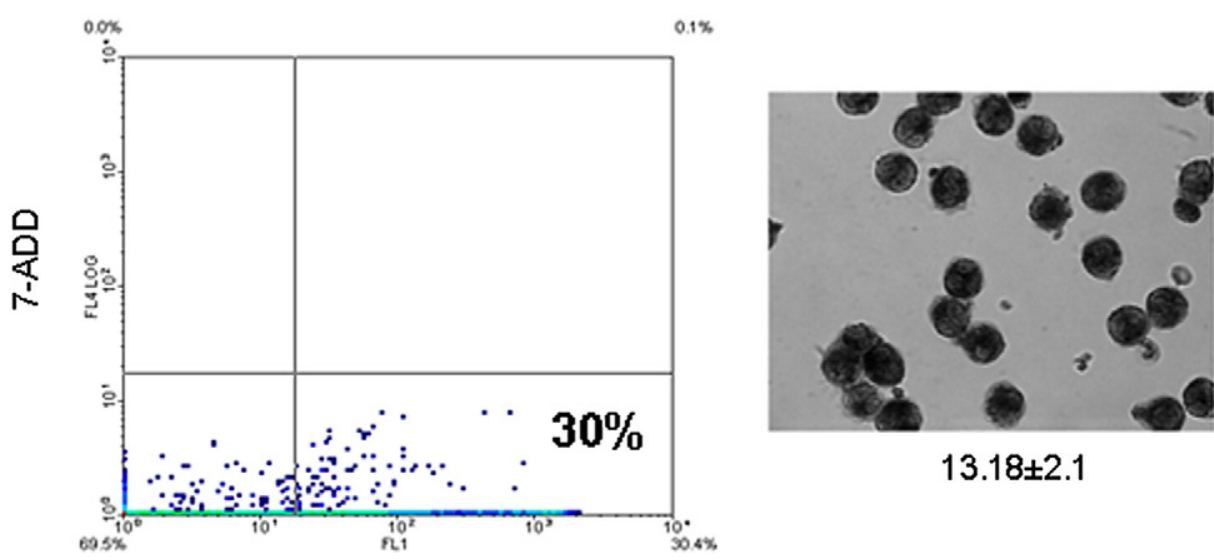

$13.18 \pm 2.1$

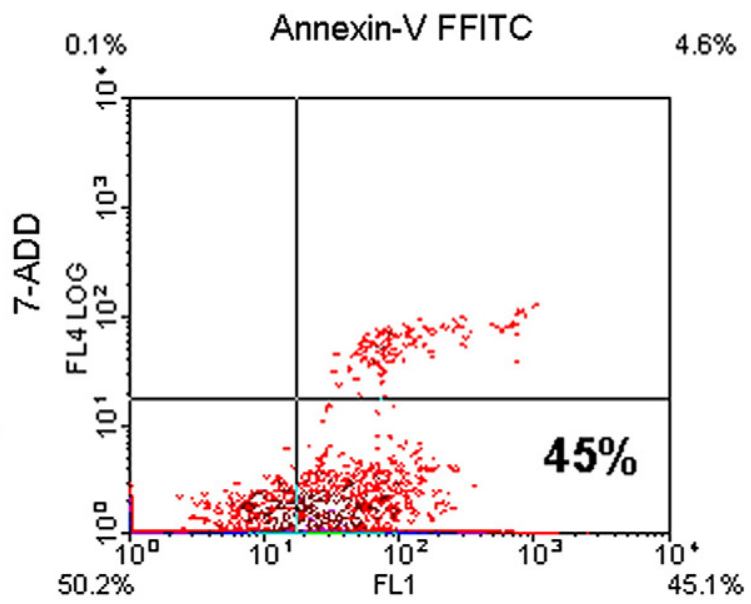

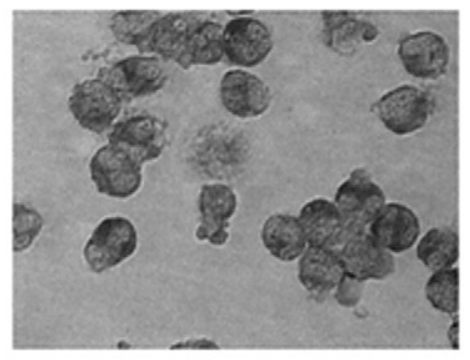

$46.7 \pm 3.7$

Annexin-V FFITC

Fig. 1 Representative results of neutrophil apoptosis assessed by 7-ADD and annexin V-FITC staining in a patient with sepsis and a healthy volunteer. Photograph representing neutrophil apoptosis with May-Grünwald-Giemsa staining. Right side of figure: cells that stained positive for annexin V-FITC and negative for 7-ADD were in the early stages of apoptosis (lower right quadrant; $45.1 \%$ patient with sepsis [A]; $15.5 \%$ control [B]). Cells staining positive for both annexin V-FITC and 7-ADD were either in the later stages of apoptosis or necrotic (upper right quadrant). Cells negative for both were viable and not undergoing apoptosis (lower left quadrant). Results are expressed as a percentage in each quadrant. Left side of figure: annexin V-FITC-positive cells, healthy volunteer (A). B, Patient with sepsis. Photograph: contains media data values of apoptosis neutrophils in septic patients (A) vs in healthy volunteer (B). May-Grünwald-Giemsa-stained cells showed shrinkage, nuclear coalescence, and nuclear chromatin condensation.

between $9 \%$ and $28 \%$, whereas the frequency in healthy subjects was between $50 \%$ and $70 \%[8,11,18,19]$.

The null hypothesis of this study was that the levels of neutrophil apoptosis in patients with septic shock are not equivalent to those in healthy controls at the end of their clinical evolution (12 days). For this study, a less than $20 \%$ difference in the level of neutrophil apoptosis between healthy controls and patients with septic shock was taken as an alternative hypothesis. Based on this assumption, for a single-tailed test with an error probability of $5 \%$ and a power of $80 \%$, a sample size of 20 healthy controls and 80 patients with septic shock was established.

\subsection{Data analysis}

To test the null hypothesis that the level of neutrophil apoptosis is different in healthy controls and patients with septic shock, the 95\% confidence interval (CI) for the difference between the mean apoptosis values (end point for the septic shock patients and baseline-unique value for controls) was calculated. Analyses were similarly performed for 28-day survivors and nonsurvivors.

To explore whether baseline apoptosis values can predict 28-day mortality, a Cox regression model was applied to the data, where the dependent variable was the time of death and the independent variables were baseline apoptosis values, therapeutic interventions (activated protein $\mathrm{C}$ therapy, appropriate antibiotic therapy, low-dose steroid therapy, insulin therapy directed at maintaining a blood glucose level $<150 \mathrm{mg} / \mathrm{dL}$ blood), disease severity measures (admission APACHE II score and admission SOFA score), sex, and age. The multivariate analysis of 28day survival was based on the standard Cox continuous proportional hazards model using a stepwise approach [20]. 
The explanatory variables were used in a categorized or dichotomized form.

The relative hazard $\left(\mathrm{RR}=\exp \left(\beta_{i}\right)\right)$ was used as a measure of the risk of death in different groups, where $\beta_{i}$ is the basic parameter in the Cox model. Kaplan-Meier estimates of mortality, along with risk ratios and $95 \%$ CIs were used to describe the relative risk of death. These curves were compared using the log-rank test.

Multiple linear regression models were used to assess the association of the percentage of neutrophil apoptosis from the onset of sepsis with the following variables: age, sex, indexes of severity (SOFA, APACHE II, lactate level, $\mathrm{ScvO}_{2}$ ), pharmacologic treatment of sepsis (blood glucose level, lowdose steroid therapy, activated protein $\mathrm{C}$ therapy, appropriate antibiotic therapy), procalcitonin, and bacteriemia.

Data are presented as mean \pm SD. Statistical analyses were performed using the SPSS software program (version 15.0, Chicago, IL). We used the $\chi^{2}$ test and Fisher exact test, to compare categorical variables, and the Student $t$ test, to compare continuous variables, as appropriate.

The difference from baseline and between the groups was evaluated by 2 -way analysis of variance for repeated

Table 1 Characteristics of the study population

\begin{tabular}{|c|c|c|c|}
\hline Characteristics & Nonsurvivors, $\mathrm{n}=43$ & Survivors, $\mathrm{n}=37$ & $P$ \\
\hline Age $(y)$, mean \pm SD & $70.1 \pm 11.4$ & $66.9 \pm 13.6$ & .26 \\
\hline Sex, M/F, n (\%) & $28(53.8) / 15(35.4)$ & $24(53.6) / 13(46.4)$ & 1.00 \\
\hline \multicolumn{4}{|l|}{ Prior or preexisting conditions, $\mathrm{n}(\%)$} \\
\hline Hypertension & $22(51.2)$ & $19(51.3)$ & 1.00 \\
\hline Coronary artery disease & $5(11.6)$ & $6(16.2)$ & .53 \\
\hline Other heart disease & $10(23.2)$ & $6(16.2)$ & .57 \\
\hline Congestive heart failure & $8(18.6)$ & $4(10.8)$ & .36 \\
\hline Diabetes mellitus & $12(27.9)$ & $9(24.3)$ & .80 \\
\hline Chronic pulmonary disease & $7(16.3)$ & $13(35.1)$ & .06 \\
\hline Liver disease & $7(16.3)$ & $5(13.5)$ & 1.00 \\
\hline Cancer & $8(18.6)$ & $9(24.3)$ & .58 \\
\hline Chronic renal failure & $11(25.6)$ & $3(8.1)$ & .07 \\
\hline \multicolumn{4}{|l|}{ Admission category, n (\%) } \\
\hline Medical & $10(23.2)$ & $10(27.0)$ & .47 \\
\hline Elective surgery & $27(62.8)$ & $33(89.2)$ & .95 \\
\hline Emergency surgery & $24(55.8)$ & $18(48.6)$ & .65 \\
\hline \multicolumn{4}{|l|}{ Type surgery } \\
\hline Cardiac surgery & $10(23.3)$ & 7 (18.9) & .64 \\
\hline Abdominal surgery or peritonitis & $18(41.9)$ & $15(40.5)$ & .90 \\
\hline Cerebral trauma or brain surgery & $1(2.3)$ & $1(2.7)$ & .91 \\
\hline Thoracic surgery & $0(0.0)$ & $1(2.7)$ & .28 \\
\hline Vascular surgery & $3(6.9)$ & $2(5.4)$ & .77 \\
\hline Others surgery & $0(0.0)$ & $3(8.1)$ & .06 \\
\hline \multicolumn{4}{|l|}{ Severity of septic shock } \\
\hline APACHE II points, mean \pm SD & $24.9 \pm 5.1$ & $21.1 \pm 5.2$ & .03 \\
\hline SOFA points, mean \pm SD & $9.8 \pm 2.6$ & $8.9 \pm 2.9$ & .16 \\
\hline $\mathrm{ARDS}$, mean $\pm \mathrm{SD}$ & $40(93)$ & $32(86.5)$ & .52 \\
\hline Arterial lactate $(\mathrm{mEq} / \mathrm{L})$, mean $\pm \mathrm{SD}$ & $3.7 \pm 3.5$ & $3.1 \pm 2.1$ & .43 \\
\hline $\mathrm{CRP}(\mathrm{mg} / \mathrm{L})$, mean $\pm \mathrm{SD}$ & $249.9 \pm 123.6$ & $269.8 \pm 125.9$ & .50 \\
\hline $\mathrm{ScvO}_{2}(\%)$ & $76.3(11.0)$ & $77.6(10.8)$ & .44 \\
\hline Procalcitonin $(\mathrm{ng} / \mathrm{mL})$, mean $\pm \mathrm{SD}$ & $28.6 \pm 45.1$ & $48.7 \pm 73.4$ & .18 \\
\hline Use of vasopressors, $\mathrm{n}(\%)$ & $43(100)$ & $37(100)$ & 1.00 \\
\hline Time to extubation (d) & $15.1 \pm 14.2$ & $19.9 \pm 14.2$ & .13 \\
\hline Leukocyte count $\left(\right.$ cells $\left./ \mathrm{mm}^{3}\right)$ at $24 \mathrm{~h}$, mean $\pm \mathrm{SD}$ & $18480.7 \pm 8436.2$ & $18606.9 \pm 9472.6$ & .95 \\
\hline Leukocyte count (cells $\left./ \mathrm{mm}^{3}\right)$ at $5 \mathrm{~d}$, mean $\pm \mathrm{SD}$ & $17930.3 \pm 9889.9$ & $15536.8 \pm 7482.3$ & .29 \\
\hline Leukocyte count (cells $/ \mathrm{mm}^{3}$ ) at $12 \mathrm{~d}$, mean $\pm \mathrm{SD}$ & $19441.9 \pm 11028.9$ & $13822.6 \pm 5877.9$ & .04 \\
\hline Length of ICU stay $(d)$, mean \pm SD & $23.3 \pm 25.0$ & $16 . \pm 5.4$ & .000 \\
\hline Length of hospital stay $(\mathrm{d})$, mean $\pm \mathrm{SD}$ & $28.2 \pm 20.0$ & $16.4 \pm 13.6$ & .003 \\
\hline \multicolumn{4}{|c|}{ Specific strategies for pharmacologic treatment in sepsis, $n(\%)$} \\
\hline Maintain blood glucose level <150 mg/dL, n (\%) & $28(65.1)$ & $13(35.1)$ & .02 \\
\hline Low-dose steroid therapy, n (\%) & $24(55.8)$ & $16(43.2)$ & .64 \\
\hline Activated protein $\mathrm{C}$ therapy, $\mathrm{n}(\%)$ & $13(30.2)$ & $8(21.6)$ & .61 \\
\hline Appropriate antibiotic therapy, $\mathrm{n}(\%)$ & $18(41.9)$ & $27(72.9)$ & .004 \\
\hline
\end{tabular}

ARDS indicates acute respiratory distress syndrome. $P \leq .05$ was considered significant. 
measurements (analysis of variance followed by Scheffe test). Correlation analysis between variables was performed using Pearson correlation coefficient. A probability value of $P \leq .05$ was considered significant.

\section{Results}

\subsection{Patient demographics and clinical characteristics}

Table 1 shows the characteristics of the septic shock patients and their distribution according to 28-day mortality. At baseline, the demographics and severity of septic shock were similar in the 28-day nonsurvivor group $(n=43)$ and the 28-day survivor group $(\mathrm{n}=37)$. Notably, in the nonsurviving group, the APACHE score $(P=.03)$, length of ICU stay $(P=.0001)$, length of hospital stay $(P=.003)$, and leukocyte count at 12 days $(P=$ $.04)$ were increased compared with survivors, whereas the number of patients undergoing appropriate antibiotic therapy $(P=.004)$ or receiving insulin therapy to maintain blood glucose less than $150 \mathrm{mg} / \mathrm{dL}(P=.02)$ was less (Table 1$)$.

The lungs and the abdomen were the most common sites of infection, occurring in $50.0 \%$ and $26.2 \%$ of the patients in the 2 groups, respectively (Table 2). The incidence of grampositive, gram-negative infections and fungus was similar in the 2 groups (Table 2).

\subsection{Neutrophil apoptosis}

A significant decrease in neutrophil apoptosis (annexin VFITC positive and 7-ADD negative) was observed in septic shock patients at 24 hours, 5 days, and 12 days after diagnosis $(14.8 \% \pm 13.4 \%, 13.4 \% \pm 8.4 \%$, and $15.4 \% \pm$ $12.8 \%$, respectively) compared with healthy volunteers $(37.6 \% \pm 12.8 \% ; P<.0001 ;$ Fig. 2$)$. No significant difference in neutrophil apoptosis was observed between 28-day nonsurvivors and survivors $(P>.05$; Fig. 3$)$.

\subsection{Correlation percentage of neutrophil apoptosis and plasma neutrophil levels}

Correlation analysis provided no evidence that the percentage of neutrophil apoptosis at 24 hours, 5 days, and 12 days from the start of septic shock is related to the plasma neutrophil level in surviving patients (24 hours, $r=0.09, P=$ $.592 ; 5$ days, $r=0.37, P=.88 ; 12$ days, $r=0.48, P=.12$ ) or nonsurviving patients (24 hours, $r=0.08, P=.58 ; 5$ days, $r=0.40, P=.01 ; 12$ days, $r=0.08, P=.70)$.

\subsection{8-day mortality and apoptosis}

The mortality rate at 28 days was $53.7 \%$ (43/80) with an in-hospital mortality of $68.7 \%(55 / 80)$. The crude hazard ratio (HR) of mortality for patients with septic

Table 2 Sites of infection and types of microorganisms causing infection in patients with septic shock

\begin{tabular}{|c|c|c|c|c|}
\hline Variable & Total, $\mathrm{n}=80$ & Nonsurvivors, $\mathrm{n}=43$ & Survivors, $\mathrm{n}=37$ & $P$ \\
\hline \multicolumn{5}{|l|}{ Site of infection, ${ }^{a} \mathrm{n}(\%)$} \\
\hline Lung & $40(50.0)$ & $19(44.2)$ & $21(56.7)$ & .21 \\
\hline Intra-abdominal & $21(26.2)$ & $15(34.8)$ & $6(16.2)$ & .20 \\
\hline Urinary tract & $7(8.7)$ & $2(4.6)$ & $5(13.5)$ & .16 \\
\hline Other ${ }^{b}$ & $12(15.0)$ & $5(11.6)$ & $7(18.9)$ & .36 \\
\hline Positive blood culture & $34(42.5)$ & $19(44.2)$ & $15(40.5)$ & .74 \\
\hline \multicolumn{5}{|l|}{ Type of organism, ${ }^{\mathrm{c}} \mathrm{n}(\%)$} \\
\hline Gram-positive cocci & $31(38.7)$ & $17(39.5)$ & $14(37.8)$ & .30 \\
\hline Aureus staphylococcus & $9(11.2)$ & $5(11.6)$ & $4(10.8)$ & .91 \\
\hline Methicillin resistant & $6(7.5)$ & $4(9.3)$ & $2(5.4)$ & .51 \\
\hline Methicillin susceptible & $3(3.7)$ & $1(2.3)$ & $2(5.4)$ & .47 \\
\hline Epidermidis staphylococcus & $8(10.0)$ & $4(9.3)$ & $4(10.8)$ & .82 \\
\hline Streptococcus species & $5(6.2)$ & $3(7.0)$ & $2(5.4)$ & .77 \\
\hline Enterococcus species & $9(11.2)$ & $5(11.6)$ & $4(10.8)$ & .91 \\
\hline Gram-negative bacilli & $55(68.7)$ & $26(60.5)$ & $29(78.4)$ & .08 \\
\hline Pseudomonas aeruginosa & $9(11.2)$ & $3(6.9)$ & $6(16.2)$ & .11 \\
\hline Klebsiella pneumoniae & $15(18.7)$ & $7(16.3)$ & $8(21.6)$ & .50 \\
\hline Acinetobacter baumannii & $4(5.0)$ & $3(6.9)$ & $1(2.7)$ & .39 \\
\hline Other gram-negative & $27(33.7)$ & $13(30.2)$ & $14(37.8)$ & .41 \\
\hline Fungus & $15(18.7)$ & $8(18.6)$ & 7 (18.9) & .92 \\
\hline Candida albicans & $8(10.0)$ & $5(11.6)$ & $3(8.1)$ & .60 \\
\hline Other Candida species & $7(8.7)$ & $3(6.9)$ & $4(10.8)$ & .55 \\
\hline
\end{tabular}

$P \leq .05$ was considered significant.

${ }^{a}$ The site of infection was either documented or presumed based on clinical findings.

${ }^{\mathrm{b}}$ Other sites of infection included the blood, skin, central nervous system, bones and joints, cardiac system, and reproductive organs

${ }^{c}$ Patients may have had more than 1 organism cultured 


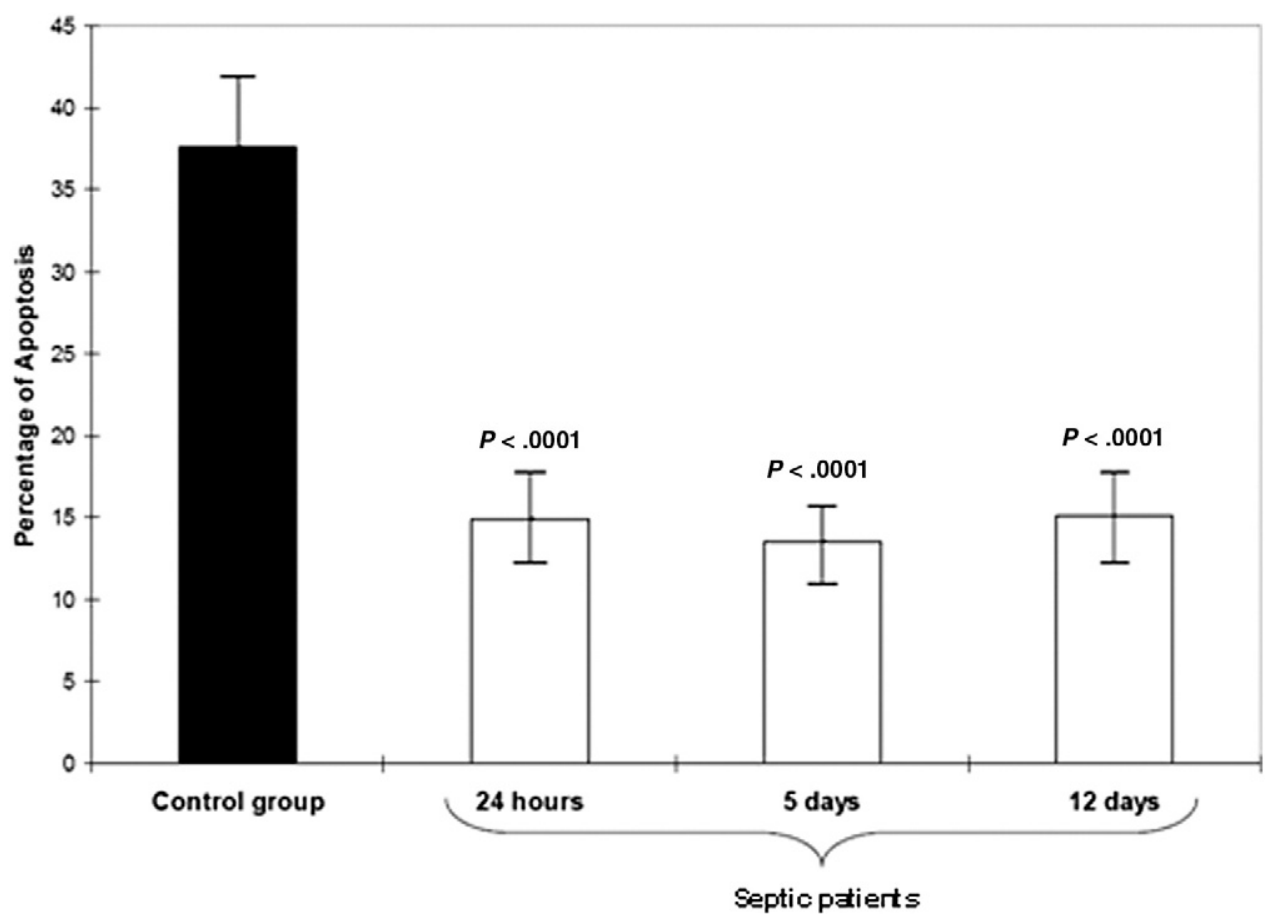

Fig. 2 Comparison of the mean percentage of neutrophil apoptosis between healthy controls (dark bars) and patients in septic shock (light bars) at different sampling times after the start of septic shock. Significant differences were observed between the control group and septic patients at the 3 time points studied.

shock who survived to day 28 did not differ from nonsurvivors according to the mean percentage of apoptosis at baseline (at 24 hours, HR, 1.006; 95\% CI, 0.98-1.03; $P=.60)$.
After performing the Cox multivariant analysis adjustment for factors (Table 3), the mean percentage of apoptosis measured at 24 hours did not stand out as an independent mortality risk factor (HR, 1.004; 95\% CI, 0.96-1.04;

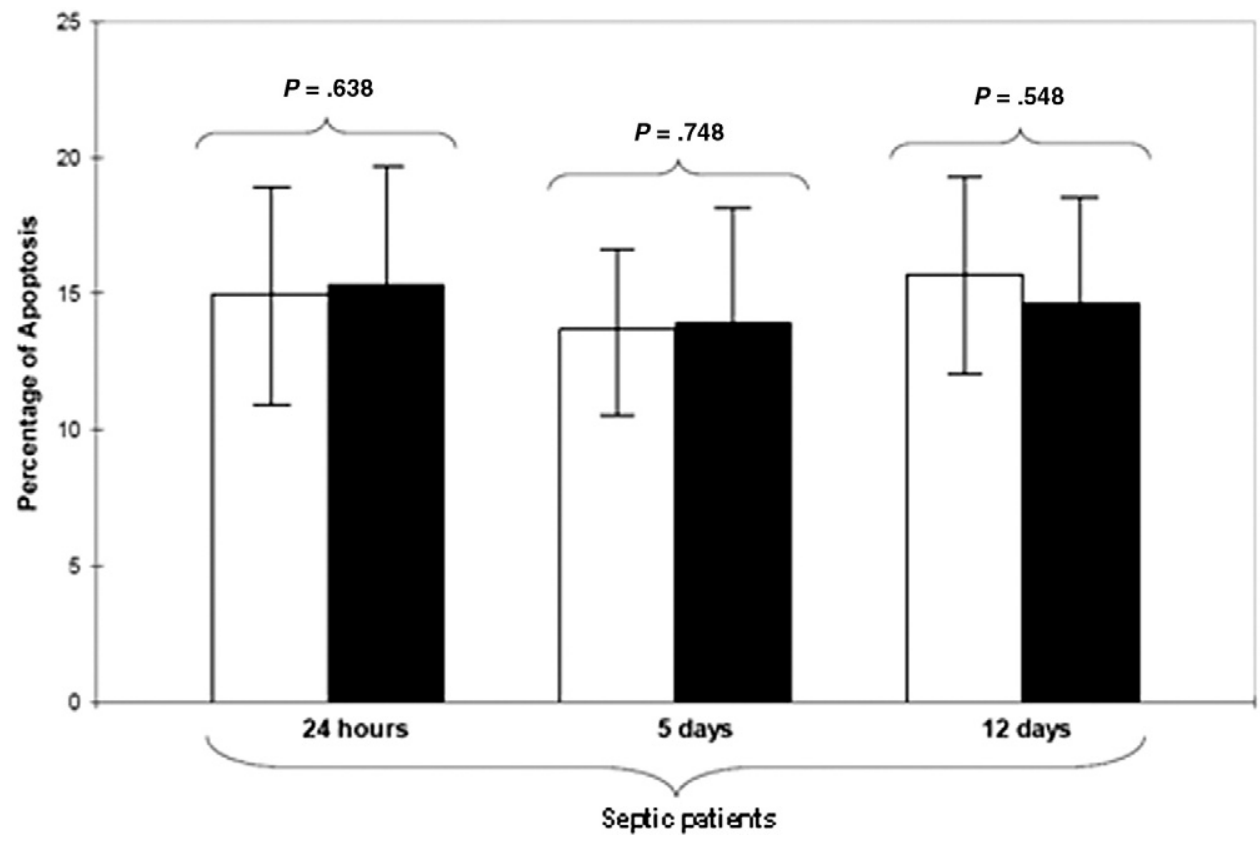

Fig. 3 Comparison of the mean percentage of neutrophil apoptosis between 28-day nonsurviving (light bars) and surviving septic shock patients (dark bars) at different sampling times after the start of septic shock. No significant differences were observed between the 2 groups at the 3 time points studied. 
Table 3 Independent variables selected by the stepwise Cox regression analysis model as associated with 28-day ICU mortality

\begin{tabular}{llc}
\hline Characteristics & $P$ & $\begin{array}{l}\text { Adjusted HR } \\
(95.0 \% \mathrm{CI})\end{array}$ \\
\hline Sex (female, 1) & .95 & $0.98(0.45-2.11)$ \\
Age (y) & .47 & $1.01(0.97-1.05)$ \\
APACHE II & .04 & $1.13(1.003-1.28)$ \\
SOFA & .63 & $0.95(0.78-1.15)$ \\
Blood glucose level $<150 \mathrm{mg} / \mathrm{dL}$ & .51 & $0.99(0.98-1.008)$ \\
Low-dose steroid therapy & .38 & $1.40(0.65-3.03)$ \\
Activated protein C therapy & .30 & $1.48(0.70-3.11)$ \\
Appropriate antibiotic therapy & .001 & $3.46(1.64-7.26)$ \\
Apoptosis & .83 & $1.004(0.96-1.04)$ \\
\hline$P \leq .05$ was considered significant. & &
\end{tabular}

$P=.83)$. The only identifiable independent mortality risk factors were appropriate antibiotic therapy and the APACHE II score. Thus, the mortality rate at 28 days was 3.46 times greater $(95 \% \mathrm{CI}, 1.64-7.26 ; P=.001)$ in patients who received an incorrect antibiotic treatment compared with patients who received the correct treatment. The mortality rate was also greater with a greater APACHE II score (adjusted HR, 1.13; 95\% CI, 1.002-1.28; $P=.04$ ).

\subsection{Determinant apoptosis factors}

Analyzing, by means of multiple linear regression, the effect of the different factors on the percentage of neutrophil apoptosis, from day 1 of the onset of sepsis, revealed that age was inversely associated with apoptosis $(\beta=.40 ; t=2.34 ; \quad P=.02 ; 95 \%$ CI, 0.06-0.93), whereas blood glucose level $(\beta=.44 ; t=2.52 ; P=.01$; $95 \% \mathrm{CI}, 0.01-0.12)$ and lactate level $(\beta=.48 ; t=2.36$; $P=.02 ; 95 \% \mathrm{CI}, 0.05-0.79)$ were positively associated with it (Table 4).

\section{Discussion}

The 2 most relevant findings of this study are that neutrophil apoptosis is decreased in patients with septic shock for at least 12 days with no significant differences between 28-day nonsurvivors and survivors and that neutrophil apoptosis is not an independent predictor of mortality risk. In addition, it was observed that apoptosis was greater in relationship with higher levels of blood glucose level and lactate level and lower as age increased.

The term apoptosis (from the Greek "fall of the leaves") was introduced by Kerr et al [21] in 1971 to describe a physiologic cell death distinct from necrosis or pathologic cell death. In recent years, the study of apoptosis in sepsis has gained interest due to the fact that it can help explain part of the enigmatic pathophysiology of this illness. Most studies carried out along these lines come from preclinical research, using experimental models of sepsis, that are often based on lethal bacterial toxin-based studies, monospecific intravenous microbial challenge, or pretreatment approaches, which do not replicate septic patients' status adequately in clinical practice [10].

Delayed neutrophil apoptosis has been documented in animals and septic patients only during the first 24 hours after the diagnosis of sepsis $[18,22,23]$. Guo et al [23] demonstrated that neutrophils in septic mice survive longer compared with nonseptic mice [23]. In septic patients, the decrease in neutrophil apoptosis was also documented by Taneja et al [18], reporting the percent neutrophil apoptosis in healthy controls as $52 \% \pm 7.8 \%$ compared with $6.2 \% \pm$ $1.1 \%$ in septic patients; Martins et al [19], reporting the median neutrophil apoptosis in healthy controls as $11.3 \%$ vs $9.1 \%$ in septic patients; Härter et al [8], reporting a median percent neutrophil apoptosis of $64 \%$ in healthy controls vs $28.8 \%$ in septic patients; and Fialkow et al [11], who reported a median percent neutrophil apoptosis in healthy controls of $69 \% \pm 1.1 \%$ vs $38 \% \pm 3.7 \%$ in septic patients. To

Table 4 Multiple linear regression analyses showing the relationship between variables and apoptosis

\begin{tabular}{llllll}
\hline Variable & SE & $\beta$ & $t$ & $P$ & $95 \%$ CI \\
\hline Constant & 35.41 & & -2.97 & .006 & $(-178.17,-32.60)$ \\
Age (y) & 0.21 & 0.40 & 2.34 & .02 & $(0.06,0.93)$ \\
Sex & 4.22 & 0.22 & 1.51 & .14 & $(-2.26,15.10)$ \\
SOFA & 1.04 & 0.12 & 0.75 & .45 & $(-1.35,2.93)$ \\
APACHE II & 0.57 & 0.17 & 0.86 & .39 & $(-0.68,1.68)$ \\
Lactate level & 0.17 & 0.48 & 2.36 & .02 & $(0.05,0.79)$ \\
ScvO $_{2}$ & 0.20 & 0.14 & 0.83 & .41 & $(-0.25,0.60)$ \\
Procalcitonin & 0.09 & 0.31 & 1.67 & .10 & $(-0.03,0.35)$ \\
Blood glucose level & 0.02 & 0.44 & 2.52 & .01 & $(0.01,0.12)$ \\
Low-dose steroid therapy & 4.47 & 0.09 & 0.58 & .56 & $(-6.57,11.84)$ \\
Activated protein C therapy & 4.57 & 0.07 & 0.47 & .64 & .26 \\
Appropriate antibiotic therapy & 4.49 & 0.18 & 1.14 & $.4 .24,11.55)$ & $(-4.08,14.37)$ \\
Bacteriemia & 4.56 & 0.13 & 0.80 & .43 & $(-5.72,13.03)$ \\
\hline
\end{tabular}

$\beta$ indicates standardized regression coefficient; $t$, corresponding $t$ values. $P \leq .05$ was considered significant. 
the contrary of that previously stated, our results confirm decreased neutrophil apoptosis not only during the first 24 hours after the onset of sepsis but also that this reduction is maintained over a period of at least 12 days.

In the present study, we detected no differences in neutrophil apoptosis between nonsurviving and surviving patients. This finding may be due to the fact that septic shock is the most serious form of sepsis and produces a more intense inhibition of apoptosis [11]. Consequently, the return to normal values of apoptosis in surviving patients happens later. Similarly, the present study found no correlation between decreased plasma neutrophil apoptosis and leukocytosis in surviving and nonsurviving patients. This fact is surprising because an important mechanism of leukocytosis that can be observed in septic shock is explained by the stimulation of leukocyte production in the bone marrow [24]. On the other hand, we did observe lower blood leukocyte levels in surviving patients on day 12 of septic shock.

The 28-day mortality observed in the present study was similar to that reported in other studies including patients with the same clinical risk [25]. The patients included in our study had an average APACHE score of 23.2 and SOFA score of 9.4, and Cox multiple regression analysis did not show that the mean percentage of apoptosis is an independent predictor of mortality in patients with septic shock. We must point out that, of the independent variables selected by Cox regression analysis, we only identified correct antibiotic treatment as an independent risk factor for mortality. This result strengthens the recommendations of the Surviving Sepsis Campaign [26], which showed that correct, early, wide-spectrum antibiotherapy is a fundamental pillar in handling sepsis.

Different factors have been cited as being able to influence neutrophil apoptosis (age, blood glucose level, corticoids, hypoxia, tobacco, etc) [27,28]; however, they have not been analyzed in patients with sepsis. In our study, carried out on patients with sepsis, we observed greater neutrophil apoptosis in those with higher levels of blood glucose level and lactate level and lesser as age increased. These findings are concordant with those observed in medical literature and described under different conditions $[27,28]$. We believe that, as this study was not designed to clarify these issues, other studies should be carried out aimed at analyzing them in more detail.

The present study has 2 main limitations. First, the evaluation of neutrophil apoptosis was carried out during the first 12 days after the onset of the clinical symptoms of septic shock. However, the study lasting until the normalization of apoptosis would possibly have been of interest. Furthermore, because apoptosis occurs via a complex signaling cascade that is tightly regulated at multiple points, there are many opportunities to evaluate the activity of the proteins involved (cytomorphological alterations, DNA fragmentation, detection of caspases, membrane alterations, detection of apoptosis in whole mounts, mitochondrial assays) [29]. There are a large variety of assays available, but each assay has advantages and disadvantages [29]. We have used annexin V-FITC labeling to measure apoptosis. The advantage is sensitivity (it can detect a single apoptotic cell). The disadvantage is that the membranes of necrotic cells are labeled as well. To avoid that and to demonstrate the membrane integrity of the phosphatidylserine-positive cells, neutrophils were stained simultaneously with 7-ADD and in parallel with May-Grünwald-Giemsa. The cells that showed shrinkage, nuclear coalescence, and nuclear chromatin condensation were considered as apoptotic.

\section{Conclusions}

In conclusion, over the first 12 days of septic shock development, the level of neutrophil apoptosis decreased and did not return to normal values, with no difference between surviving and nonsurviving patients. Moreover, the determinant factors for apoptosis levels were age, blood glucose level, and lactate level. Consequently, we concluded that apoptosis in sepsis is a phenomenon that requires further study to clearly determine its role before proposing that new medical treatments for sepsis have antiapoptotic effects. 


\section{Appendix A. Summary of inclusion criteria}

\begin{tabular}{|c|c|c|}
\hline Infection criteria & Modified SIRS criteria & For dysfunctional organs or systems * \\
\hline $\begin{array}{l}\text { Patients had to have a known infection or a } \\
\text { suspected infection, as evidenced by one } \\
\text { or more of the following: white cells in a } \\
\text { normally sterile body fluid; perforated } \\
\text { viscus; radiographic evidence of } \\
\text { pneumonia in association with the } \\
\text { production of purulent sputum; a } \\
\text { syndrome associated with a high risk of } \\
\text { infection (eg, ascending cholangitis) }\end{array}$ & $\begin{array}{l}\text { Patients had to meet at least } 3 \text { of the following } \\
4 \text { criteria: a core temperature of } \geq 38^{\circ} \mathrm{C} \\
\left(100.4^{\circ} \mathrm{F}\right) \text { or } \leq 36^{\circ} \mathrm{C}\left(96.8^{\circ} \mathrm{F}\right) \text {; a heart rate of } \\
\geq 90 \text { beats per min, except in patients with a } \\
\text { medical condition known to increase the heart } \\
\text { rate or those receiving treatment that would } \\
\text { prevent tachycardia; a respiratory rate of } \geq 20 \\
\text { breaths per min or a Paco } 2 \text { of } \leq 32 \mathrm{~mm} \mathrm{Hg} \text { or } \\
\text { the use of mechanical ventilation for an acute } \\
\text { respiratory process; a white cell count of } \\
\geq 12000 / \mathrm{mm}^{3} \text { or } \leq 4000 / \mathrm{mm}^{3} \text { or a } \\
\text { differential count showing }>10 \% \text { immature } \\
\text { neutrophils }\end{array}$ & $\begin{array}{l}\text { Patients had to meet at least } 1 \text { of the } \\
\text { following } 5 \text { criteria: for cardiovascular } \\
\text { system dysfunction, the arterial systolic } \\
\text { blood pressure had to be } \leq 90 \mathrm{~mm} \mathrm{Hg} \text { or the } \\
\text { mean arterial pressure } \leq 70 \mathrm{~mm} \mathrm{Hg} \text { for at } \\
\text { least } 1 \text { hour despite adequate fluid } \\
\text { resuscitation, adequate intravascular volume } \\
\text { status, or the use of vasopressors in an } \\
\text { attempt to maintain a systolic blood pressure } \\
\text { of } \geq 90 \mathrm{~mm} \mathrm{Hg} \text { or a mean arterial pressure } \\
\text { of } \geq 70 \mathrm{~mm} \mathrm{Hg} \text {; for kidney dysfunction, } \\
\text { urine output had to be }<0.5 \mathrm{~mL} / \mathrm{kg} \text { of body } \\
\text { weight per hour for } 1 \text { hour, despite adequate } \\
\text { fluid resuscitation; for respiratory system } \\
\text { dysfunction, the ratio of Pao } 2 \text { to Fio } 2 \text { had to } \\
\text { be } \leq 250 \text { in the presence of other } \\
\text { dysfunctional organs or systems or } \leq 200 \text { if } \\
\text { the lung was the only dysfunctional organ; } \\
\text { for hematologic dysfunction, the platelet } \\
\text { count had to be }<80000 / \mathrm{mm}^{3} \text { or to have } \\
\text { decreased by } 50 \% \text { in the } 3 \text { days preceding } \\
\text { enrollment; in the case of unexplained } \\
\text { metabolic acidosis, the pH had to be } \leq 7.30 \\
\text { or the base deficit had to be } \geq 5.0 \text { mmol } / \mathrm{L} \text { in } \\
\text { association with a plasma lactate level that } \\
\text { was }>1.5 \text { times the upper limit of the normal } \\
\text { value for the reporting laboratory }\end{array}$ \\
\hline
\end{tabular}

SIRS denotes systemic inflammatory response syndrome; $\mathrm{PaCO}_{2}$, partial pressure of arterial carbon dioxide; $\mathrm{PaO}_{2}$, partial pressure of arterial oxygen; and $\mathrm{FiO}_{2}$, fraction of inspired oxygen.

* The first sepsis-induced organ or system dysfunction had to develop within 24 hours before study enrollment.

\section{Appendix B. Patients and healthy volunteers}

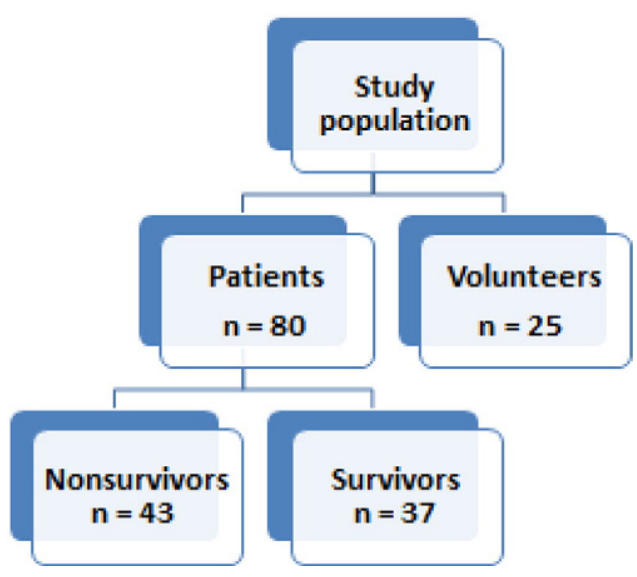

\section{Appendix C. Supplementary data}

Supplementary data to this article can be found online at doi:10.1016/j.jcrc.2011.09.001.

\section{References}

[1] Angus DC, Linde-Zwirble WT, Lidicker J, et al. Epidemiology of severe sepsis in the United States: analysis of incidence, outcome, and associated costs of care. Crit Care Med 2001;29:1303-10.

[2] Esteban A, Frutos-Vivar F, Ferguson ND, et al. Sepsis incidence and outcome: contrasting the intensive care unit with the hospital ward Crit Care Med 2007;35:1284-9.

[3] Brun-Buisson C, Meshaka P, Pinton P. EPISEPSIS Study. Intensive Care Med 2004;30:580-8.

[4] Marshall JC, Cook DJ, Christou NV, et al. Multiple organ dysfunction score: a reliable descriptor of a complex clinical outcome. Crit Care Med 1995;23:1638-52.

[5] Tamayo E, Gualis J, Florez S, et al. Comparative study of single-dose and 24-hour multiple-dose antibiotic prophylaxis for cardiac surgery. J Thorac Cardiovasc Surg 2008;136:1522-7.

[6] Ertel W, Keel M, Infanger M, et al. Circulating mediators in serum of injured patients with septic complications inhibit neutrophil apoptosis 
through up-regulation of protein-tyrosine phosphorylation. J Trauma 1998;44:767-77.

[7] Bone RC, Balk RA, Cerra FB, et al. Definition for sepsis and organ failure and guidelines for the use of innovative therapies in sepsis. Chest 1992;101:1644-55.

[8] Härter L, Mica L, Stocker R, et al. Mcl-1 correlates with reduced apoptosis in neutrophils from patients with sepsis. J Am Coll Surg 2003;197:964-73.

[9] Marshall JC, Watson RW. Apoptosis in the resolution of systemic inflammation. In: Vincent JL, editor. Yearbook of intensive care and emergency medicine. Berlin: Springer-Verlag; 1997.

[10] Hotchkiss RS, Karl IE. The pathophysiology and treatment of sepsis. N Engl J Med 2003;348:138-48.

[11] Fialkow L, Fochesatto Filho L, Bozzetti MC, et al. Neutrophil apoptosis: a marker of disease severity in sepsis and sepsis-induced acute respiratory distress syndrome. Crit Care 2006;10:R155.

[12] Wesche DE, Lomas-Neira JL, Perl M, et al. Leukocyte apoptosis and its significance in sepsis and shock. J Leukoc Biol 2005;78:325-37.

[13] Rivers E, Nguyen B, Havstad S, et al. Early goal-directed therapy in the treatment of severe sepsis and septic shock. N Engl J Med 2001;345:1368-77.

[14] Ferreira FL, Bota DP, Bross A, et al. Serial evaluation of the SOFA score to predict outcome in critically ill patients. JAMA 2001;286: 1754-8.

[15] Fonteriz RI, Sánchez A, Mollinedo F, et al. The role of intracellular acidification in calcium mobilization in human neutrophils. Biochem Biophys Acta 1991;1093:1-6.

[16] Knaus WA, Draper EA, Wagner DP, et al. APACHE II: a severity of disease classification system. Crit Care Med 1985;13:818-29.

[17] Moreno R, Vincent JL, Matos R, et al. The use of maximum SOFA score to quantify organ dysfunction/failure in intensive care. Results of a prospective, multicentre study. Working Group on Sepsis related Problems of the ESICM. Intensive Care Med 1999;25:686-96.
[18] Taneja R, Parodo J, Jia SH, et al. Delayed neutrophil apoptosis in sepsis is associated with maintenance of mitochondrial transmembrane potential and reduced caspase-9 activity. Crit Care Med 2004;32: $1460-6$.

[19] Martins PS, Kallas EG, Neto MC, et al. Upregulation of reactive oxygen species generation and phagocytosis and increased apoptosis in human neutrophils during severe sepsis and septic shock. SHOCK 2003;20:208-12.

[20] Cox DR. Regression models and life-tables. J Stat Soc B 1972;34: 187-220.

[21] Kerr JF, Willye AH, Curie AR. Apoptosis: a basis biological phenomenon with wideranging implications in tissue kinetics. $\mathrm{Br} \mathrm{J}$ Cancer 1972;26:239-57.

[22] Jimenez MF, Watson RW, Parodo J, et al. Dysregulated expression of neutrophil apoptosis in the systemic inflammatory response syndrome. Arch Surg 1997;132:1263-70.

[23] Guo RF, Sun L, Gao H, et al. In vivo regulation of neutrophil apoptosis by C5a during sepsis. Leukoc Biol 2006;80:1575-83.

[24] Smith JA. Neutrophils, host defense, and inflammation: a doubleedged sword. J Leukoc Biol 1994;56:672-86.

[25] Kumar A, Roberts D, Wood KE, et al. Duration of hypotension before initiation of effective antimicrobial therapy is the critical determinant of survival in human septic shock. Crit Care Med 2006;34:1589-96.

[26] Dellinger RP, Levy MM, Carlet JM, et al. Surviving sepsis campaign: international guidelines for management of severe sepsis and septic shock. Intensive Care Med 2008;34:17-60.

[27] Duffin R, Leitch AE, Fox S, et al. Targeting granulocyte apoptosis: mechanisms, models, and therapies. Immunol Rev 2010;236:28-40.

[28] Fülöp Jr T, Fouquet C, Allaire P, et al. Changes in apoptosis of human polymorphonuclear granulocytes with aging. Mech Ageing Dev 1997;1-3:15-34.

[29] Elmore S. Apoptosis: a review of programmed cell death. Toxicol Pathol 2007;35:495-516. 